\title{
Morphology and classification of atrioventricular defects
}

\author{
GIAN PIERO PICCOLI ${ }^{1}$, LEON M. GERLIS, JAMES L. WILKINSON, \\ KAROLYI LOZSADI, FERGUS J. MACARTNEY ${ }^{2}$, AND ROBERT H. \\ ANDERSON ${ }^{2}$ \\ From the Department of Paediatrics, Cardiothoracic Institute, Brompton Hospital, London; \\ Department of Pathology, Grimsby General Hospital, South Humberside; Institute of Child Health, \\ Alder Hey Children's Hospital, Liverpool; Department of Paediatric Surgery, Semmelzveis Medical \\ University, Budapest, Hungary; and Thoracic Unit, The Hospital for Sick Children, London
}

SUMMARY Anatomical studies were made on 114 necropsy specimens of atrioventricular defects with atrioventricular concordance. The malformation is characterised by disproportion between the ventricular inlet and outlet dimensions and a malorientation of the aortic valve relative to the atrioventricular valve or valves. Associated with this there is a characteristic 'scooped-out' appearance of the muscular ventricular septum, gross abnormalities of the membranous components of the septum as compared with the normal heart, and narrowing of the aortic outflow tract. Hearts with these anatomical features can be divided into partial and complete forms depending on the morphology of the atrioventricular annuli. In the partial form the septal leaflets are conjoined to give separate mitral and tricuspid orifices, the conjoined leaflets being displaced into the ventricles and usually attached to the crest of the septum. In the complete form, anterior and posterior components of the 'septal' leaflets are separate, so that a single valve orifice connects the atrial to the ventricular chambers. Further subdivision of the complete form, apart from the morphology of the anterior leaflet, is dependent upon the presence or absence of an ostium primum atrial septal defect.

Many papers have been published on the varying morphology of atrioventricular defects ('atrioventricular canal malformations') (Rogers and Edwards, 1948; Wakai and Edwards, 1956, 1958; Van Mierop et al., 1962; Frater, 1965; Rastelli et al., 1966; Van Mierop and Alley 1966; Rastelli et al., 1967; Goor et al., 1968; Rastelli et al., 1968; Bharati and Lev, 1973; Tenckhoff and Stamm, 1973; Goor and Lillehei, 1975; Ugarte et al., 1976; Van Mierop, 1977).

It is evident from those investigations that opinion is by no means unanimous concerning several important features of these anomalies. For example, there is disagreement concerning the meaning of the so-called intermediate (Wakai and Edwards, 1958) or transitional type (Wakai and Edwards, 1956; Al Omeri et al., 1965; Sumner et al., 1967; Goor and Lillehei, 1975) of atrioventricular defects.

There is disagreement as to whether a cleft between anterior and posterior components of the mitral valve (Van Mierop and Alley, 1966) or an ${ }^{1}$ G. P. P. was a visiting fellow from Ospedale GM Lancisi, Ancona, Italy.

${ }^{2}$ F. J. M. and R. H. A. are supported by the British Heart Foundation together with the Vandervell Foundation and the Joseph Levy Foundation, respectively.

Received for publication 20 December 1978 ostium primum atrial septal defect (Goor and Lillehei, 1975) are necessary for definition of an atrioventricular defect. It may be asked whether atrioventricular defects are characterised only by shortness of the inflow portion of the left ventricle (Goor et al., 1968; Tenckhoff and Stamm, 1973; Ugarte et al., 1976) or if there is also lengthening of the left ventricular outflow tract (Van Mierop et al., 1962; Gerbode et al., 1967). Finally, it may be asked what are the anatomical features that are the essence of atrioventricular defects.

We have tried to solve some of these problems by anatomical analysis of specimens catalogued as exhibiting an atrioventricular defect or one of its varieties.

\section{Subjects and methods}

We studied specimens from the pathological collections of the Brompton Hospital, London; The Hospital for Sick Children, London; the Royal Liverpool Children's Hospital, Liverpool; Grimsby General Hospital, South Humberside; and the Department of Paediatric Pathology, University of Sheffield, Sheffield.

In all the specimens, we analysed the following: 
(a) the atrioventricular fibrous annulus; (b) the relation of the inlet and outlet tracts of the left ventricle to each other, as described by Goor et al. (1968), and in relation to the distance between the lowest portion of the interventricular septum and the apex of the left ventricle; (c) the constituents of the interventricular muscular septum; (d) the left ventricular outflow tract; (e) in the presence of an anterior common leaflet, the type of attachment of its right and left ventricular components; (f) in the absence of an anterior common leaflet, the type of attachment of the anterior component of the mitral valve; and ( $\mathrm{g}$ ) the degree of fusion between the septum primum and the plane of the atrioventricular valve.

We examined only specimens with a concordant atrioventricular connection and we excluded cases

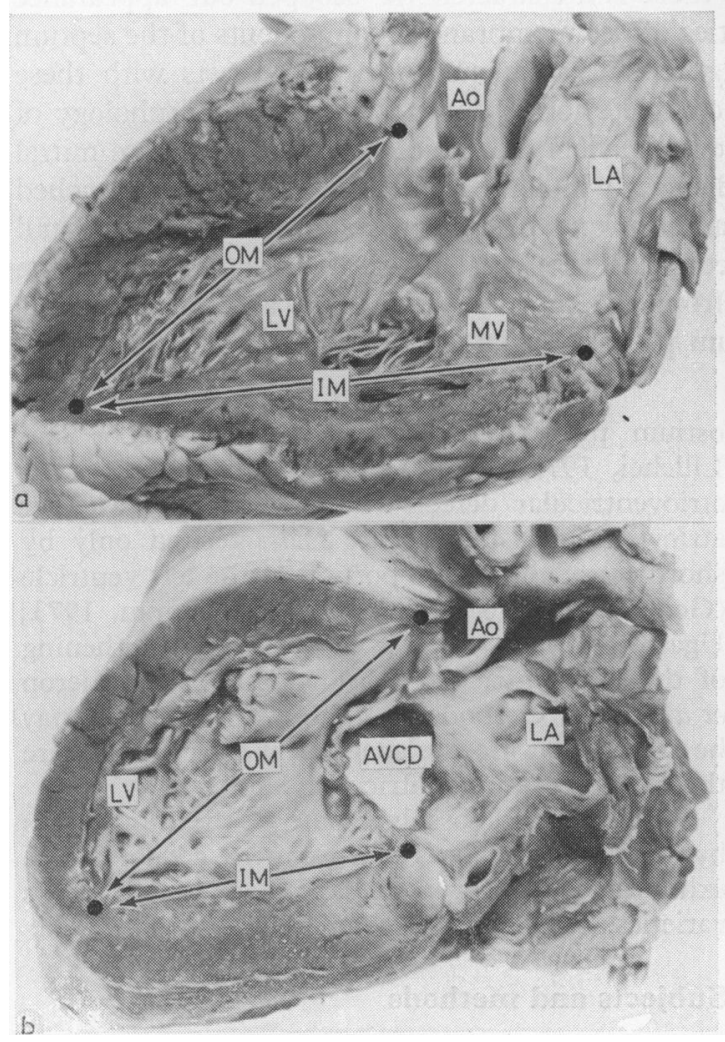

Fig. 1 Photographs of longitudinal sections of the normal left ventricle (Fig. 1a) and the left ventricle in an atrioventricular defect (Fig. 1b). The photographs show the inlet (IM) and outlet (OM) measurements taken. Note that in the atrioventricular defect there is disproportion between these measurements. ( $L A$, left atrium; $L V$, left ventricle; $M V$, mitral valve; $A o$, aorta; $A V C D$, atrioventricular canal defect). with discordant ventriculoarterial connections or single outlet of the heart. In all, 114 cases were included in our study group.

\section{Results}

In all the hearts studied, it was found that firstly the inferior edge of the atrial septum was abnormal in its attachment to the atrioventricular annulus fibrosus. Secondly, the membranous component of the cardiac septum was abnormally positioned and formed. Thirdly, the plane of the atrioventricular orifices was abnormal. Fourthly, the inlet portion of the ventricular septum was deficient (scooped-
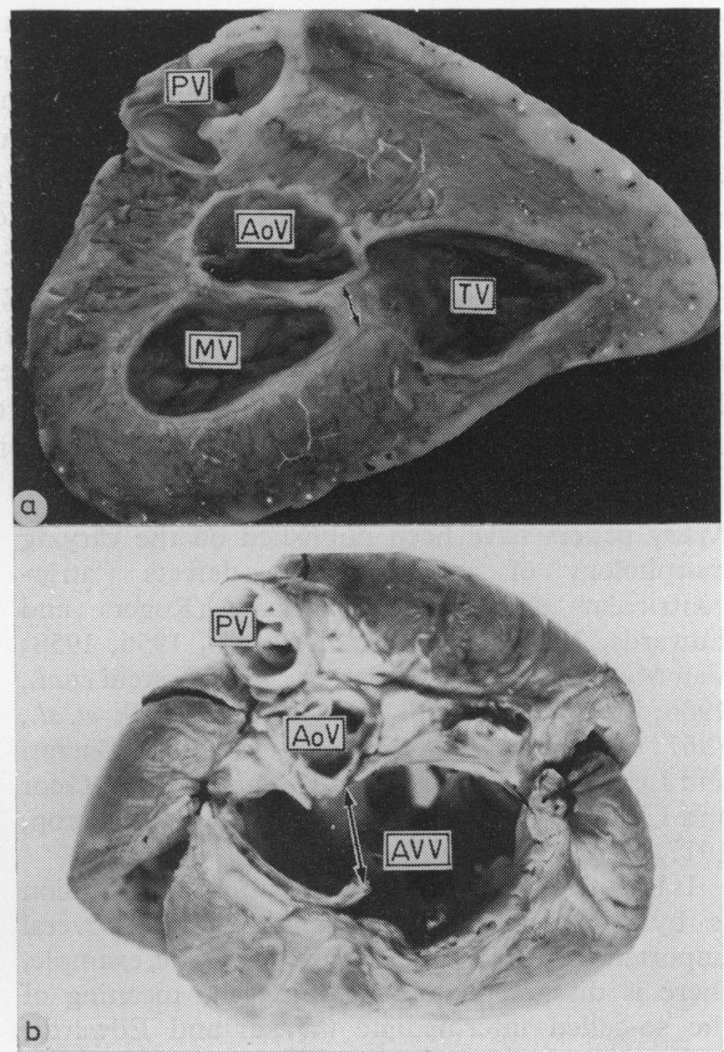

Fig. 2 Photographs of the atrioventricular junctions of a normal heart (Fig. 2a) and a heart with an atrioventricular defect (Fig. 2b). The hearts are viewed from above. Note that in Fig. 2a the aortic valve (AoV) is wedged between the mitral and tricuspid valves ( $M V$, $T V)$. There is only a short distance at the septal contiguities (arrowed). In contrast, in atrioventricular defects (Fig. 2b) the aortic valve does not occupy a wedge position and the atrioventricular orifice is 'sprung'. In the specimen illustrated there is a common atrioventricular valve $(A V V)$. (PV, pulmonary valve.) 
out) in its central portion, and, finally, the aortic valve was abnormally positioned and orientated (Fig. 1,2). There was considerable variation in the degree of formation of the septal structures and the atrioventricular valves within this pattern of disordered morphology (Table). The hearts could basically be divided into two groups: those with two discrete atrioventricular annuli and those with a common annulus.

\section{Table Atrioventricular defects}

Two divided atrioventricular annuli: partial forms

(a) Isolated cleft between anterior and posterior component of mitral valve with abnormally attached mitral valve: one case

(b) Isolated inlet ventricular septal defect with cleft between anterior and posterior component of mitral valve: one case

(c) Ostium primum atrial septal defect with abnormally attached but not clefted mitral valve: two cases

(d) Ostium primum atrial septal defect with cleft between anterior and posterior component of the mitral valve: 32 cases

with complete cleft: 26 cases

with incomplete cleft: six cases with double cleft: three cases

with partial cleft of tricuspid valve: 11 cases

(e) Ostium primum atrial septal defect with inlet ventricular septal defect but with two separate atrioventricular valves: eight cases

(i) with isolated cleft between anterior and posterior component of the mitral valve: three cases

(ii) with cleft between anterior and posterior component of mitral valve associated with cleft of septal leaflet of tricuspid valve: three cases

(iii) with isolated cleft of septal leaflet of the tricuspid valve: two cases

Common atrioventricular fibrous annulus: complete forms

(a) Ostium primum atrial septal defect confluent with inlet ventricular septal defect: 66 cases

without anterior bridging leaflet: six cases with an anterior bridging leaflet: 60 cases
HEARTS WITH TWO VALVE ANNULI

We found separate fibrous annuli of the atrioventricular valves in 44 cases. One of these hearts exhibited only an isolated cleft between the anterior and posterior component of the mitral valve. Though the atrial and ventricular septa were intact, the two components of the mitral valve were abnormally attached to the interventricular septum, along a line concave towards the atria. The atrial septal attachment was therefore similarly abnormal. The interventricular muscular septum showed the classical 'scooped-out' appearance (Fig. 3).

In one heart, a cleft between the anterior and posterior component of the mitral valve was present, without an ostium primum atrial septal defect, but with a scalloped-out appearance of the interventricular septum and a perimembranous ventricular septal defect.

In two hearts ostium primum atrial septal defects were present without any cleft between the anterior and posterior components of the mitral valve (Fig. 4). There was a lowering of the medial insertion of the mitral fibrous annulus on the superior edge of the interventricular muscular septum, which was deficient in its central portion.

In 32 hearts ostium primum atrial septal defects were present together with cleft mitral valves and the scooped-out appearance of the interventricular septum. The septal annulus separating the mitral and tricuspid septal leaflets was displaced into the ventricles and firmly attached to the crest of the septum (Fig. 5a). In 26 of the 32 cases ( $81 \%$ ) the cleft of the mitral valve was complete, that is it extended to the septal annulus. In the remaining hearts the cleft was incomplete. In three of these

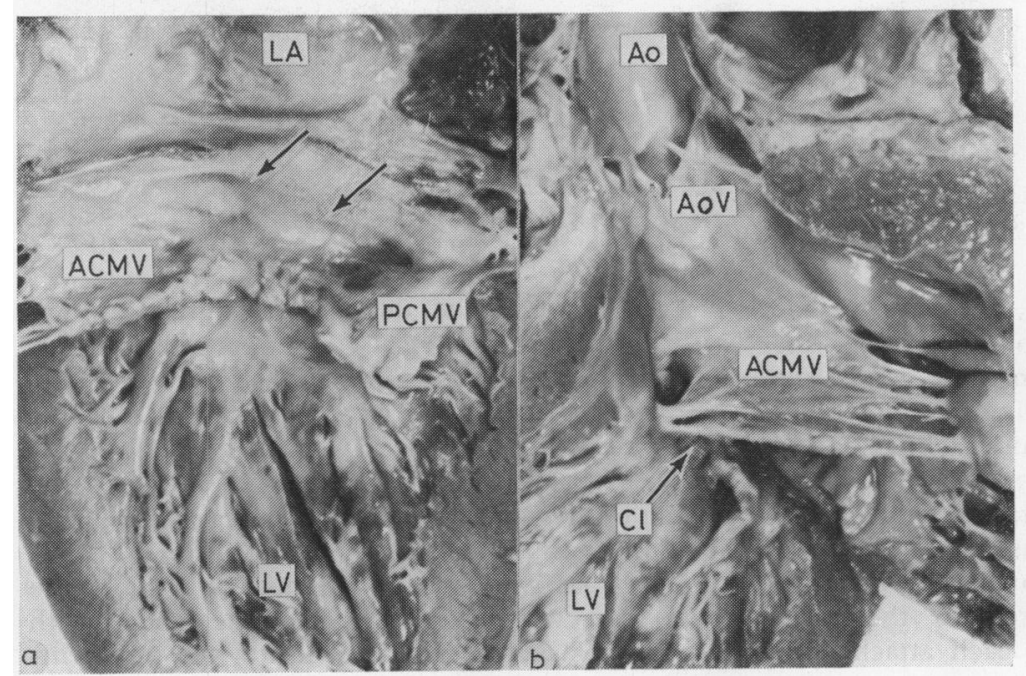

Fig. 3 Photographs showing a specimen with an isolated cleft of the mitral valve. Fig. $3 a$ shows the left atrium ( $L A$ ) leading to the left ventricle ( $L V)$. The atrial septum is intact (arrows). Note the cleft between the anterior and posterior components of the anterior leaflets of the mitral valve (ACMV, PCMV). Fig. $3 b$ shows the left ventricular outflow tract. The cleft $(\mathrm{Cl})$ is seen and the anterior component is attached to the elongated outlet septum. Other abbreviations as before. 
hearts a double cleft was present. In 11 of these hearts, the septal leaflet of the tricuspid valve was divided into discrete anterior and posterior components, the dividing notch extending to the conjoined septal annuli (Fig. 6).

In eight hearts ostium primum atrial septal defects were associated with inlet perimembranous ventricular septal defects. Of these eight hearts, three exhibited a cleft of the mitral valve alone; three had an incomplete cleft of the mitral valve together with discrete anterior and posterior components of the septal leaflet of the tricuspid valve; two did not have mitral clefts (Fig. 7), but one of these exhibited discrete components of the septal leaflet of the tricuspid valve.

\section{HEARTS WITH COMMON ATRIOVENTRICULAR ORIFICE}

Seventy cases were found with a common atrioventricular fibrous annulus (Fig. 5b, c).

In 66 of the hearts an ostium primum atrial septal defect was confluent with an inlet ventricular septal defect. the crest of the septum, the space between them being the septal crest. In the remaining 64 cases, there was a bridging anterior leaflet. In 39 of these cases the right lateral edge of the bridging anterior leaflet was attached to the superior edge of the muscular septum and, extending across the septum, to the medial papillary muscle.

In eight cases the right lateral insertion was to a 'medial' papillary muscle, displaced from its usual position towards the ventricular apex. In the remaining 17 cases the lateral attachment was to the anterolateral papillary muscle of the right ventricle. The interventricular septum showed always a 'scoopedout' appearance.

\section{COMMON FEATURES}

Measurement of the lengths of the inlet septum compared to the outlet septum (Fig. 9) showed that the outlet tract of the left ventricle was always lengthened in relation to its inlet portion. The diaphragmatic length of the inlet septum was greater than the central portion, which constituted the shortest length of the ventricular septum (Fig. 10,
Fig. 4 Photographs illustrating the morphology of an atrioventricular defect with an ostium primum atrial septal defect $(O P)$. Fig. $4 a$ shows the defect viewed from the left. There is no cleft in the mitral valve. Fig. $4 b$ shows the typical atrioventricular defect outflow tract. Abbreviations as before.

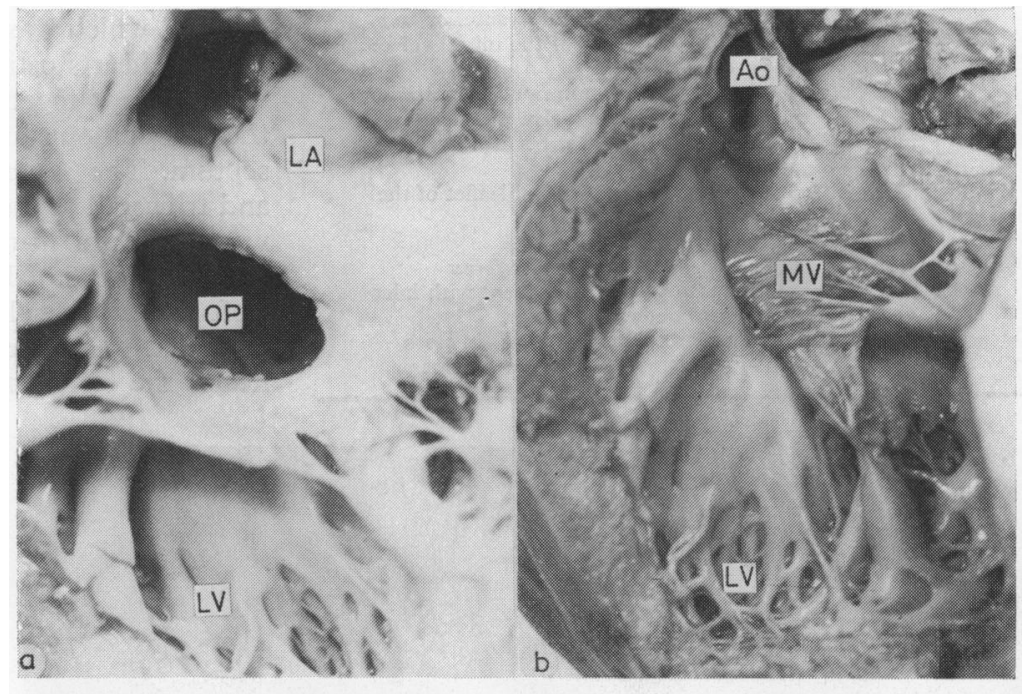

In the remaining four hearts, the edge of the atrial septum extended down to the level of the atrioventricular annulus. Thus, in these hearts, the ostium primum defect was lacking, though there was a common atrioventricular annulus (Fig. 8).

Among the cases with common annulus, six lacked a bridging anterior leaflet. In these hearts, the anterior part of the canal was guarded by two leaflets, one totally committed to the left ventricle and the other totally committed to the right ventricle. The two leaflets were each attached to
11). In general, the degree of lengthening was greater in the hearts with a common annulus than in those with separate mitral and tricuspid annuli.

In all the hearts the aortic valve was deviated anteriorly, when compared with the normal hearts, and in 90 cases it was deviated rightward (Fig. 12).

The outflow tract was narrowed as judged from anatomical observation in 68 hearts (Fig. 13).

This tract was larger than normal only in the three cases with complete defects associated with tetralogy of Fallot. (In these hearts the perimem- 


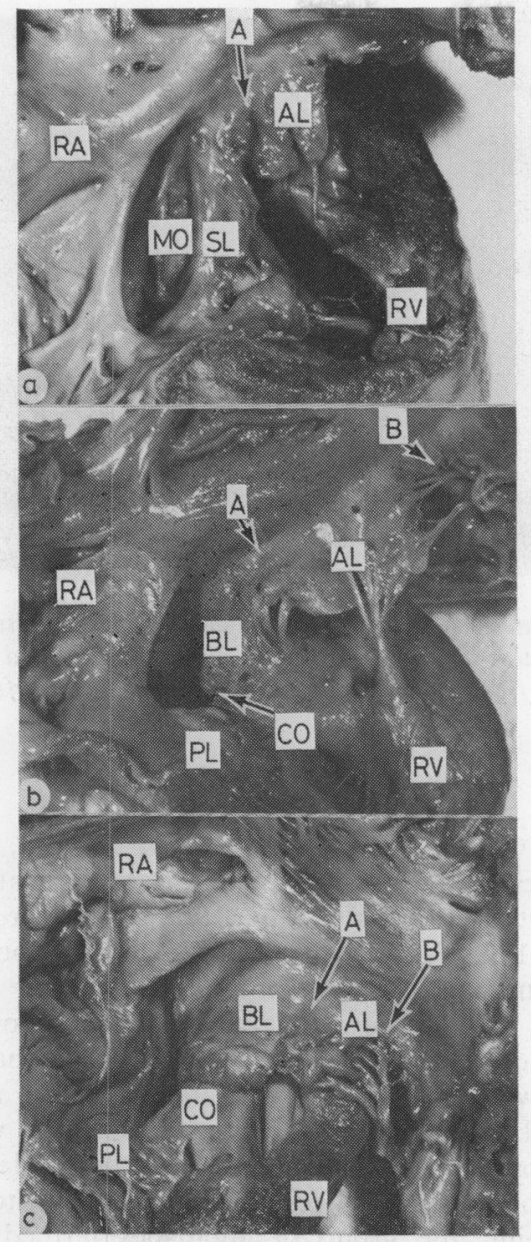

Fig. 5 Photographs illustrating the morphology of ostium primum atrioventricular defect with a cleft mitral valve (Fig. 5a) and complete atrioventricular defects (Fig. 5b, c). In Fig. 5a the opened tricuspid orifice is seen. The discrete mitral orifice (MO) is viewed through the ostium primum defect. The septal leaflets of mitral and tricuspid valves are fused both to each other and to the ventricular septum $(S L)$. The narrow septal commissure of the tricuspid valve is seen $(A)$ and is to the right of the atrial septal insertion to the annulus. It separates the septal leaflet of the tricuspid valve from the anterior leaflet $(A L)$. Fig. $5 b$ shows the comparable anatomy of a complete atrioventricular defect. However, there is now a common orifice (CO) caused by the separation of the anterior and posterior components of the septal leaflets of the atrioventricular valves. The anterosuperior commissure $(A)$ now separates the anterior bridging leaflet $(B L)$ from the anterior leaflet of the tricuspid component. This in turn is separated from the lateral leaflet by commissure B. Note also the posterior bridging leaflet (PL). Fig. $5 c$ shows a common atrioventricular defect with a greater degree of bridging of the left anterior leaflet. branous defect extended into the infundibular region because of anterior deviation of the infundibular septum. A confluent perimembranous defect therefore extended from the crux to the insertion of the infundibular septum.)

In the hearts with two annuli, in the absence of an inlet defect, an interventricular component of a membranous septum was present beneath the attachment line of the anterior component of the mitral valve to the superior edge of the interventricular muscular septum.

In the cases with inlet ventricular septal defect, the interventricular communication was bounded at the level of the infundibular septum by a remnant of the interventricular membranous septum in all the hearts with two annuli and in 48 cases $(68 \%)$ with common annulus (Fig. 14a).

A fibrous structure between the left ventricle and the right atrium was always present and was comparable with the outlet component of the atrioventricular membranous septum (Fig. 14b).

\section{Discussion}

The hallmarks of an atrioventricular defect are, firstly, a disproproportion between the inlet and outlet length of the ventricular septum and, secondly, a malorientation of the aortic valve relative to the atrioventricular valve or valves (Van Mierop et al., 1962; Van Mierop and Alley, 1966; Goor and Lillehei, 1975; Van Mierop, 1977). Atrial or ventricular septal defects and malformations of the atrioventricular valves can accompany these basic anatomical features, and the combination of these anomalies produces the variation seen in the malformation (Fig. 15).

By atrioventricular defects, therefore, we describe a continuous spectrum of malformations characterised by: (1) 'scooped-out' appearance of the interventricular septum (Campbell and Missen, 1957; Van Mierop et al., 1962; Somerville, 1966; Van Mierop and Alley, 1966; Gerbode et al., 1967; Goor et al., 1968; Tenckhoff and Stamm, 1973; Goor and Lillehei, 1975; Ugarte et al., 1976; Van Mierop, 1977; Ebert and Goor, 1978); (2) abnormal attachment of the atrioventricular leaflets to the rim of the interventricular septum (Van Mierop et al., 1962; Van Mierop and Alley, 1966; Van Mierop, 1977); (3) abnormal attachment of the inferior edge of the septum primum to the atrioventricular plane (Rogers and Edwards, 1948; Wakai and Edwards, 1956, 1958; Van Mierop et al., 1962; Van Mierop and Alley, 1966; Goor and Lillehei, 1975; Van Mierop, 1977); (4) lengthening and narrowing of the left ventricular outflow tract (Van Mierop et al., 1962; Van Mierop and Alley, 


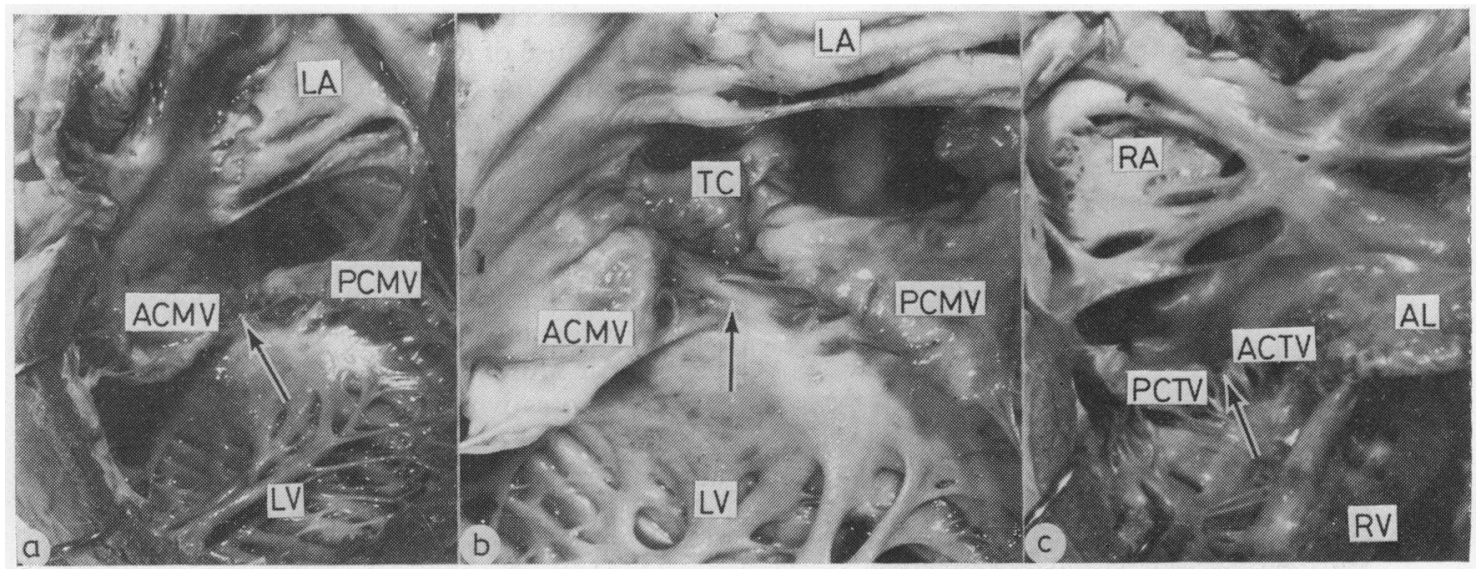

Fig. 6 Photographs illustrating a specimen with clefts between the anterior and posterior components of both mitral and tricuspid valves ( $A C M V, P C M V, A C T V, P C T V$ ) but despite this separation still with discrete mitral and tricuspid orifices. Fig. $6 a$ and $6 b$ are viewed from the left and Fig. $6 c$ from the right. The arrows point to the clefts. Other abbreviations as before.

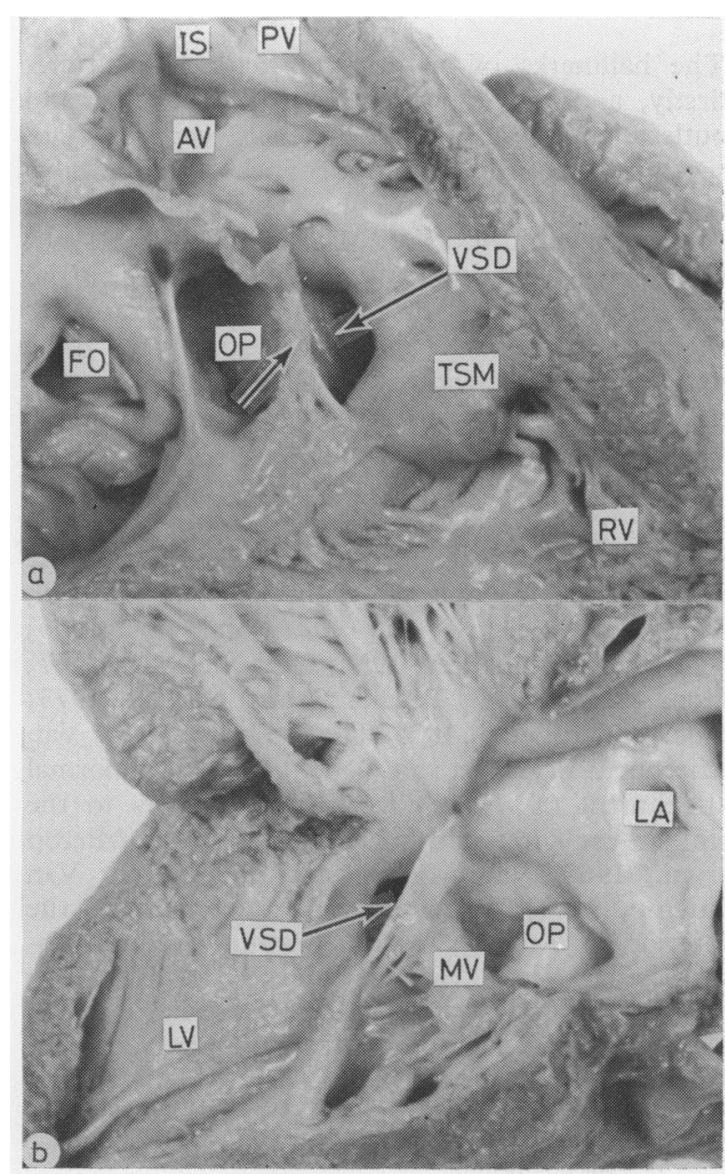

1966; Gerbode et al., 1967; Van Mierop, 1977) together with a rightward and anterior deviation of the aorta (Van Mierop et al., 1962; Van Mierop and Alley, 1966; Van Mierop, 1977); and (5) abnormal conformation of the membranous septum.

Our results show that the different anomalies can be divided into two basic constellations; namely, those with separate mitral and tricuspid annuli (partial atrioventricular defects) and those with a common atrioventricular annulus (complete defects). Our observations suggest that from the anatomical viewpoint, the group of anomalies termed transitional or intermediate types (Wakai and Edwards, 1956, 1958; Al Omeri et al., 1965; Sumner et al., 1967) are best considered as variants of the partial form in that they have discrete mitral and tricuspid annuli. It is true that the discrete annuli are in many instances produced as a consequence of a bridge of valve tissue extending across the septum from posterior to anterior leaflets and that the conjoined valves have features that resemble a common orifice (Fig. 6). It is also true that from the surgical standpoint these anomalies have features

Fig. 7 Photographs illustrating the morphology of an atrioventricular defect with an ostium primum atrial septal defect, a perimembranous inlet ventricular septal defect (VSD) but discrete mitral and tricuspid orifices without a cleft (arrows). Fig. $7 a$ is viewed from the right ventricle and shows double outlet from this ventricle; the aorta $(A V)$ and pulmonary artery (PV) being separated by the infundibular septum (IS). (FO, fossa ovalis; TSM, trabecular septomarginalis.) Fig. $7 b$ shows the left ventricular aspect. 


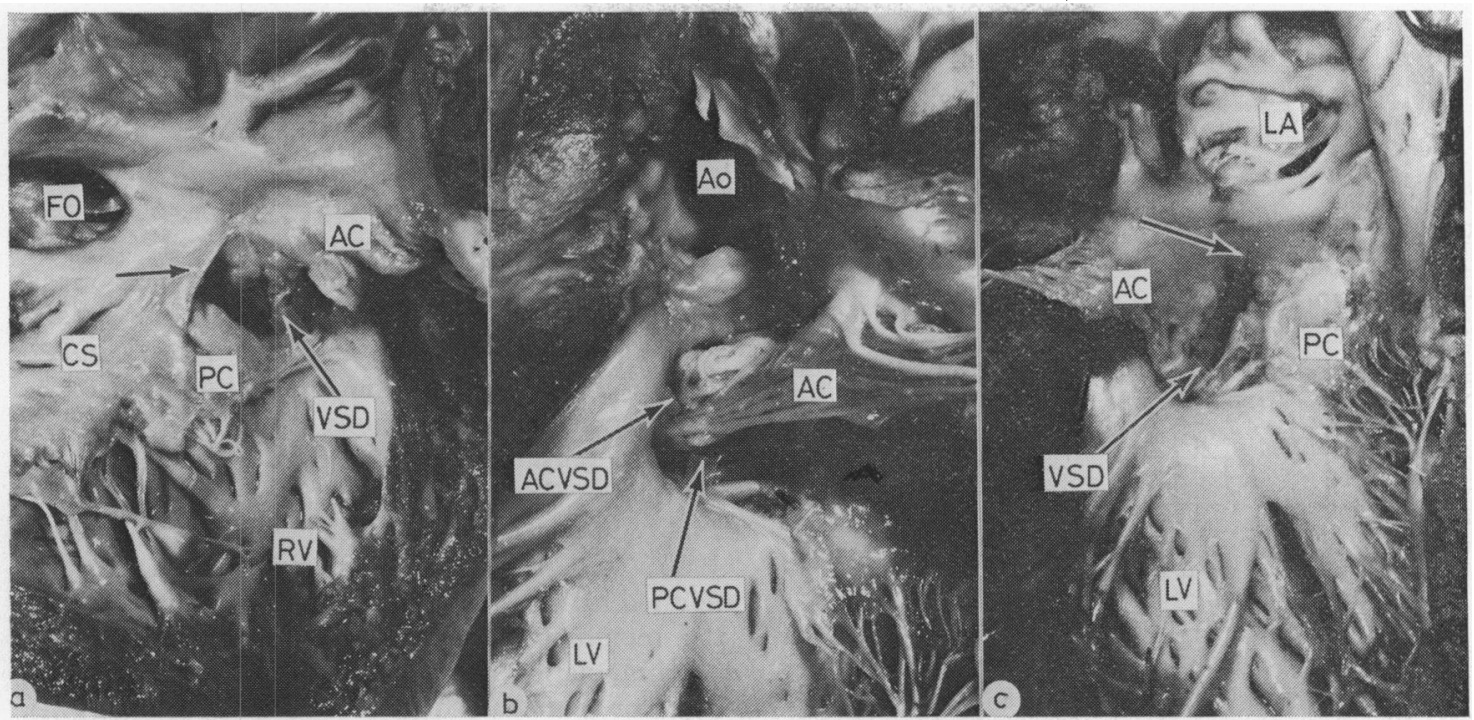

Fig. 8 Photographs illustrating the morphology of a complete atrioventricular defect without an ostium primum atrial septal defect. Fig. 8a shows the right aspect of the malformation. Both anterior and posterior leaflets (AC, PC) bridge through the ventricular septal defect to the left ventricle. Fig. $8 \mathrm{~b}$ and $8 \mathrm{c}$ show the left ventricular aspect, viewed from the outflow tract (Fig. 8b) and the inlet aspect (Fig. 8c). The anterior component of the common AV valve (AC) separates the ventricular septal defect into anterior ( $A C V S D)$ and posterior (PCVSD) components. Note that the atrial septum is intact (arrowed) and extends down to the level of the atrioventricular annulus. (CS, coronary sinus; other abbreviations as before.)

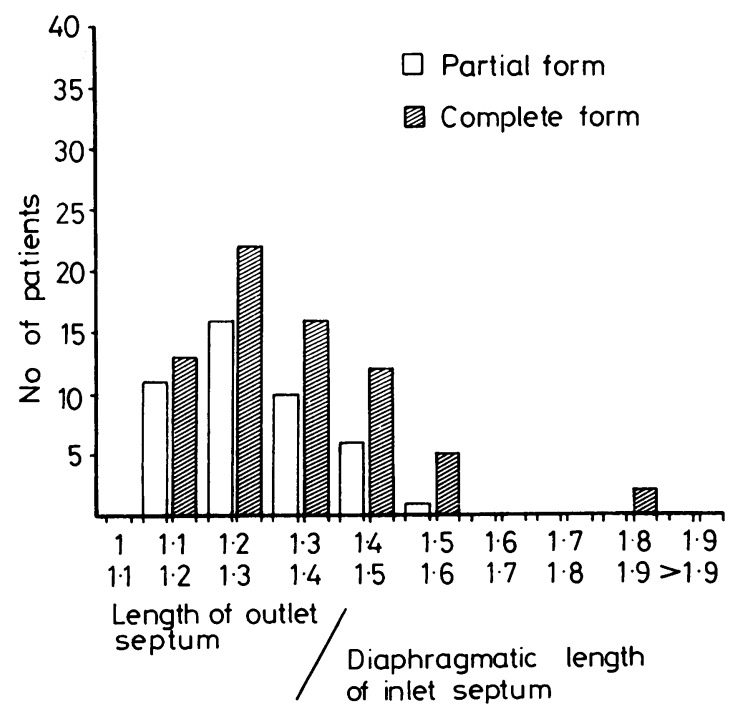

Fig. 9 Ratio between the lengths of the outlet septum and the diaphragmatic portion of the inlet septum. The length of the outlet septum was always greater than that of the diaphragmatic portion of the inlet septum. This disproportion was more evident in the complete than in the partial types of atrioventricular defects. This ratio in the normal hearts is 1 . more in common with the complete form of the malformation particularly when a large ventricular defect is present beneath the bridging leaflet tissue. None the less, to our minds the presence of bridging leaflet tissue produced two discrete annuli and permits their unequivocal categorisation on anatomical grounds as partial malformations. Indeed, we observed one heart in which an ostium primum atrial septal defect was associated with a ventricular septal defect and partitioned mitral and tricuspid annuli, in the absence of any clefts in their septal components (Fig. 7). These considerations highlight the problems engendered by usage of the adjectives 'partial' and 'complete' as applied to atrioventricular defects. We, believing we were conforming to common usage, used the terms to differentiate cases with partitioned tricuspid and mitral orifices from those with a common orifice. But it has been pointed out to us that an alternative approach (Brandt et al., 1972) is to use the term 'partial' to describe cases without a ventricular septal defect, 'complete' to describe those with a ventricular septal defect, and to use 'intermediate' in yet another way, namely to describe cases with a small ventricular septal defect (J. B. Partridge, 1979, personal communication). Thus the problems engendered by the 'intermediate' category relate to 


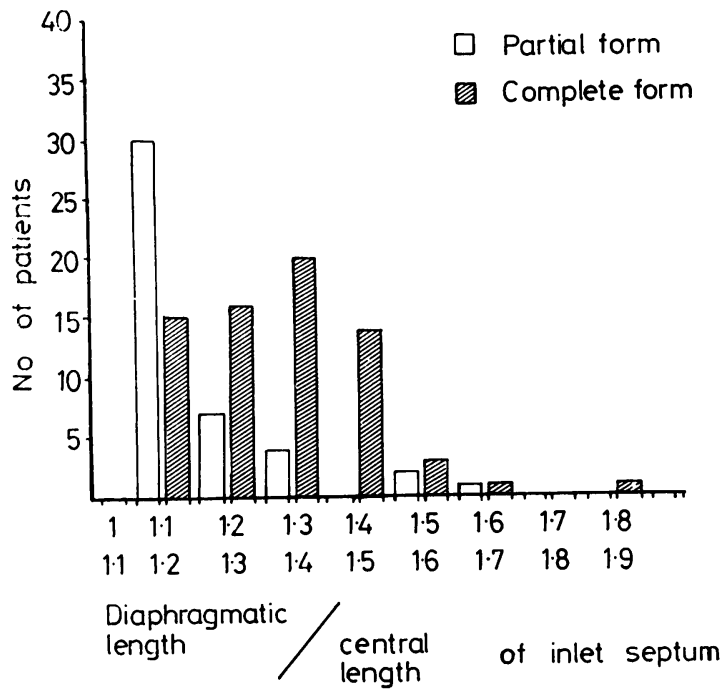

Fig. 10 Ratios between the lengths of the diaphragmatic and central portion of the inlet ventricular septum. The diaphragmatic length of the inlet septum was always greater than the central portion. This disproportion was more evident in the complete than in the partial types of atrioventricular malformations. This ratio in the normal hearts is 1.

the use of the terms 'partial' and 'complete'. In that we are using these adjectives to describe annular morphology, they are not ideal. It would be far more appropriate to divide our cases into groups with partitioned orifices (presently termed 'partial defects') and those with a common orifice (presently termed 'complete defects'). We have continued to use the terms 'partial' and 'complete' in this and our other papers (Macartney et al., 1979; Piccoli et al., 1979) in deference to what we believe is common usage, but we suggest that in future it will be better to differentiate atrioventricular defects on the basis of their annular morphology into cases with partitioned mitral and tricuspid orifices and cases with a common atrioventricular orifice.

There has been considerable discussion regarding the substrate of the disproportion of the inlet and outlet length of the ventricular septum. Many authors have indicated that there is a shortening of the diaphragmatic wall of the left ventricle (Goor et al., 1968; Tenckhoff and Stamm, 1973; Blieden et al., 1974; Ugarte et al., 1976). Yet others have indicated that the infundibular septum is excessively long (Van Mierop et al., 1962; Van Mierop and Alley, 1966; Gerbode et al., 1967). Our findings indicate that all of these factors are present (Fig. 9, $10,11)$. Measurement of the inlet septum and comparison with the length of the outlet septum do not permit deductions concerning the shortness of one or the excessive length of the other. It is more likely that a combination of these factors exists.

There has also been disagreement regarding which part of the membranous septum is missing in atrioventricular canal malformations. Some have suggested that all the membranous portion of the ventricular septum is lacking (Rogers and Edwards, 1948; Wakai and Edwards, 1956; Campbell and Missen, 1957; Wakai and Edwards, 1958; Neufeld et al., 1961). Others have suggested that the atrioventricular membranous septum together with the basal portion of the muscular ventricular septum are missing (Van Mierop et al., 1962; Al Omeri et al. 1965; Somerville, 1966; Van Mierop and Alley, 1966; Somerville and Jefferson, 1968). Yet others pointed to a deficiency in the muscular rim beneath the membranous septum which extended to the diaphragmatic wall of the left ventricle (Goor et al., 1968) or to the subaortic area (Blieden et al., 1974). Our observations substantiate the view that the crest of the inlet septum is deficient in the perimembranous area. However, we would not agree that the membranous septum is always absent.

Discussion regarding this point devolves upon definitions of the membranous septum. In all the hearts studied, the septal edge of the anterior components of the atrioventricular valves is inter-

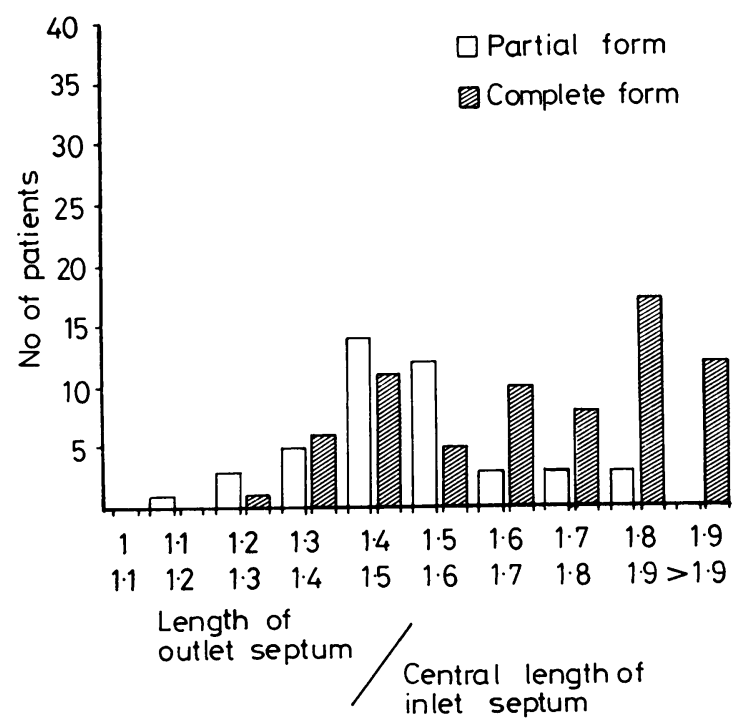

Fig. 11 Ratio between the lengths of the outlet septum and the central portion of the inlet septum. The length of the outlet septum was always greater than that of the central portion of the inlet septum. This disproportion was more evident in the complete than in the partial types of atrioventricular defects. This ratio in the normal hearts is 1 . 
posed between the left ventricular outflow tract and the right atrium. Therefore, it constitutes an atrioventricular component of a membranous septum (Fig. 8b). It occupies a different position
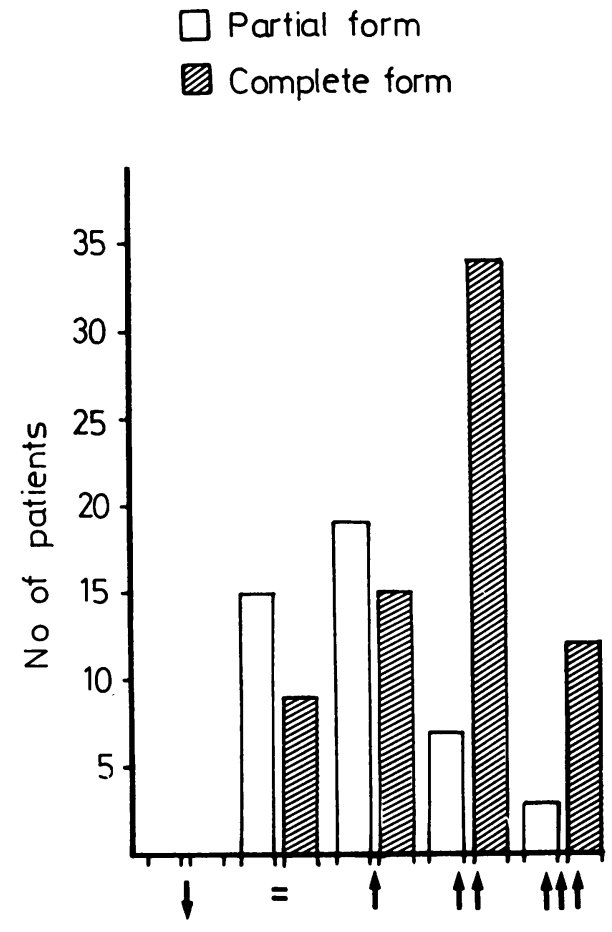

$\downarrow$ Leftward displacement of the aorta

$=$ Normal position of the aorta

f Rightward displacement of the aorta - mild

if Rightward displacement of the aorta-medium

\section{ift Rightward displacement of the} aorta - severe

Fig. 12 Rightward displacement of the aorta. This was a finding characterising 78.1 per cent of the cases. The major degrees of rightward displacement were present in the complete types of atrioventricular defects. from that found in the normal heart, being deviated anteriorly. In addition, in the malformations without perimembranous ventricular septal defect, the insertion line of the conjoined annuli of the mitral and tricuspid valves on the interventricular septum represents an interventricular membranous septum. In the malformations with perimembranous septal defect, a remnant of the interventricular component of the membranous septum was present in $\mathbf{5 7}$ cases $(73 \%)$. It must be emphasised, however, that a muscular atrioventricular septum was totally lacking in all hearts studied (Brandt et al., 1972), since the tricuspid and mitral annuli were attached to one another at the same level (Van Mierop et al., 1962; Van Mierop and Alley, 1966; Goor et al., 1968). In the normal heart the tricuspid valve is attached to the cardiac septum towards the ventri-

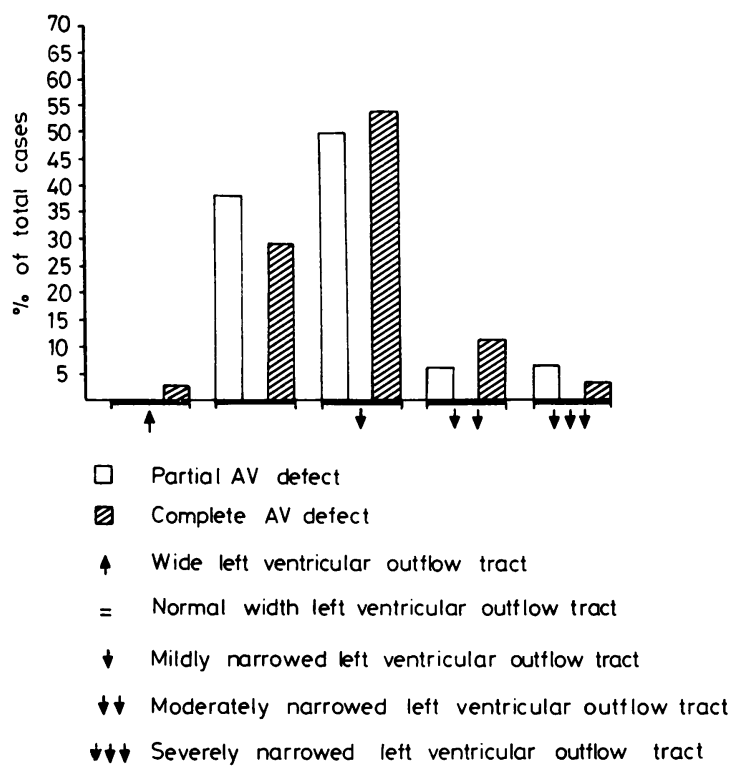

Fig. 13 Left ventricular outflow tract narrowing. This was found in 68 hearts.

cular apex as compared with the mitral valve. As a consequence of this, a small part of the membranous septum together with part of the inlet muscular septum is placed between the right atrium and the inlet part of the left ventricle. Clearly when, as in atrioventricular defects, the mitral and tricuspid annuli are attached at the same level, these atrioventricular septal components cannot exist.

We observed in our series of partial defects, two hearts in which an ostium primum defect was present without a cleft in the mitral valve. None the less, in these hearts the conjoined mitral and 
Fig. 14 Photographs of atrioventricular defects illustrating in Fig. 14a the atrioventricular membranous septum ( $A V M S$ ) and in Fig. $14 b$ an interventricular membranous septum (IVMS). Fig. $14 a$ has been prepared by sectioning the heart from the right atrium into the aortic outflow tract. (SD, septal defect; other abbreviations as before.) Fig. $14 b$ is viewed from the anterior aspect of the left ventricular outflow.

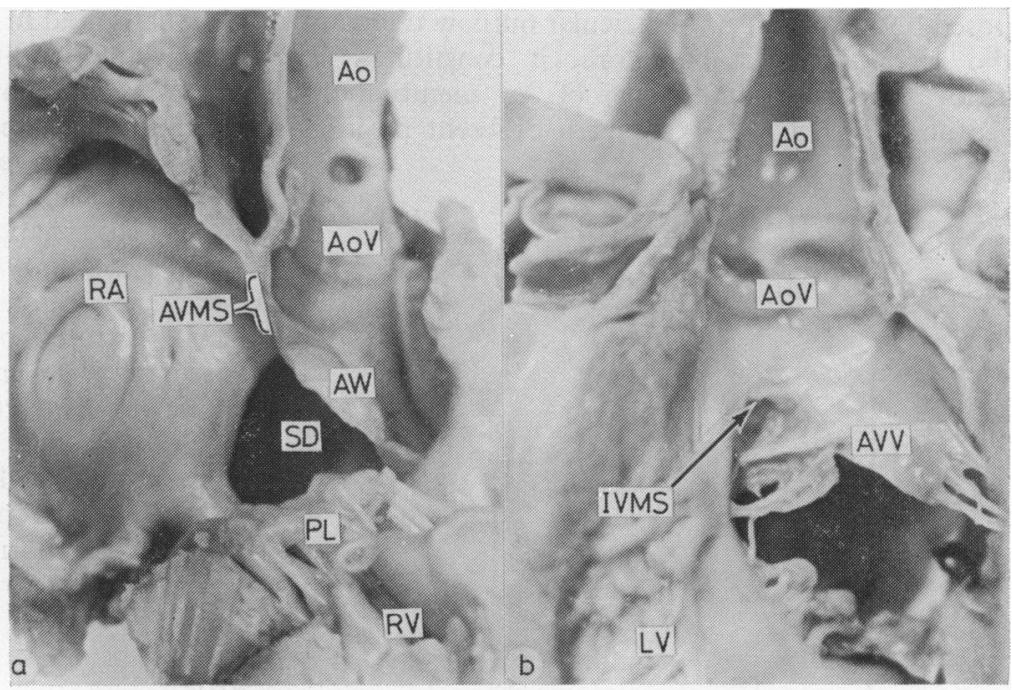

tricuspid annuli were attached to the crest of a typical 'scooped-out' ventricular septum, and the hearts had all the features of an atrioventricular defect. This type of malformation has been characterised by Goor et al. (1968) as a 'corrected' malformation since haemodynamically it resembles a secondum atrial septal defect.

In the majority of cases of the complete malformation, the atrioventricular orifices were committed in approximately equal portions between the right and left ventricle. In some hearts, however, the orifices were committed primarily to one ventricle with hypoplasia of the other ventricle. This feature was previously noted by Bharati and Lev (1973) and termed right or left ventricular dominance, and a large series of right dominance has recently been identified and described by Freedom et al. (1978). Such ventricular dominance was also observed in partial malformations in our series.

Four other cases of the complete malformation were of considerable interest. In these hearts, on first examination it appeared that the atrial septum was intact and that two annuli were formed. On closer evaluation it was seen that the anterior and posterior leaflets were continuous between right and left ventricles, a fibrous rim on the underside of the atrial septum joining together the leaflets. If we compare these cases with the diagram constructed by Goor and Lillehei (1975) for division of the atrial and ventricular components of the complete defect, these cases must be categorised as complete

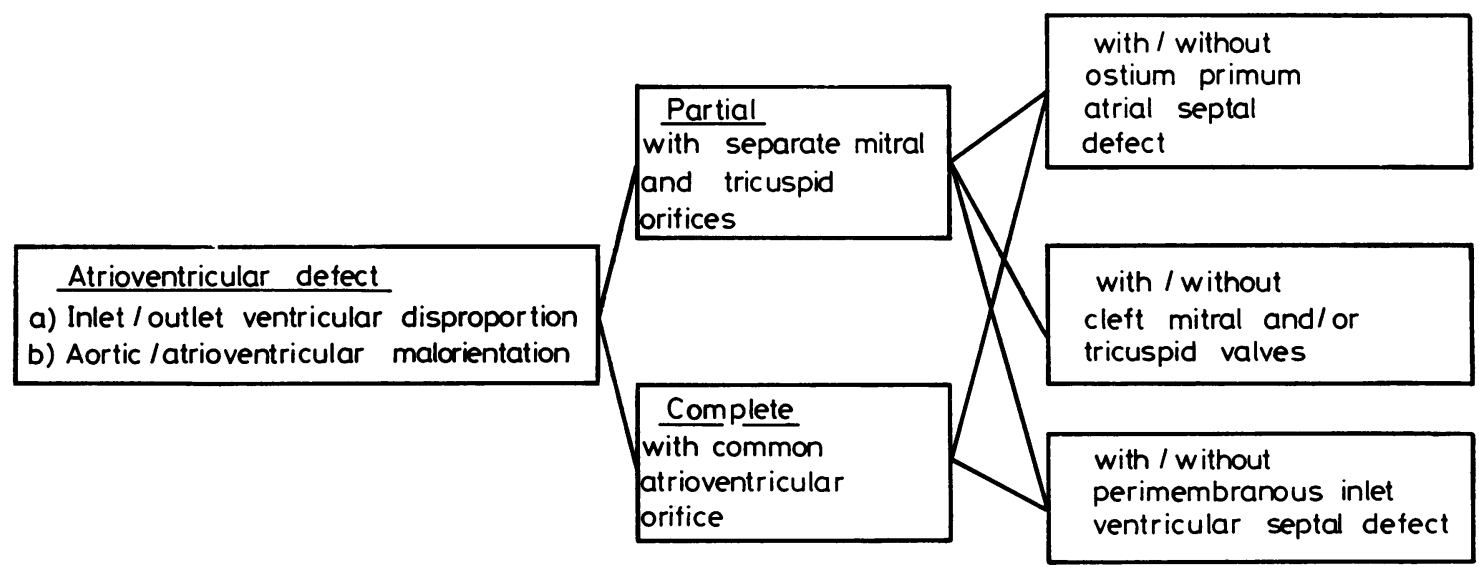

Fig. 15 Diagrammatic representation of the classification of atrioventricular defects. The complete defect always has a perimembranous inlet ventricular septal defect. 
atrioventricular defects without an ostium primum component. An alternative way of expressing this would be to say they were true atrioventricular defects, the interventricular communication being at the atrial aspect of the valve leaflets. They must be distinguished from the so-called 'atrioventricular canal ventricular septal defects' described in partial defects (Neufeld et al., 1961). In many instances, the latter defects are no more than perimembranous defects excavating the inlet portion of the ventricular septum and do not possess the stigmata of the atrioventricular defect itself (see Neufeld et al., 1961, Fig. 8). However, as with so many anomalies, there is a spectrum of malformations depending on the degree of excavation of the inlet septum. In our series of partial canal malformations, we observed a ventricular septal defect in association with a cleft mitral valve but with an intact atrial septum, this case having all the features of an atrioventricular defect. It is our opinion, therefore, that the term 'atrioventricular canal ventricular septal defect', if used at all, should be reserved for description of such cases which have the cardinal features of an atrioventricular defect.

In conclusion, our study indicates that atrioventricular defects are characterised by discrete anatomical features, namely disproportion between ventricular inlet and outlet lengths and abnormal position of the aortic valve. The other cardinal features follow from these primary abnormalities. They can be divided into partial and complete forms, depending upon whether these are separate mitral and tricuspid annuli or a common atrioventricular annulus. However, we believe that, in future, it will be more appropriate to describe these as groups with partitioned orifices or a common orifice rather than with the terms 'partial' and 'complete'. Each of these groups can be further subdivided depending on the presence or absence of an atrial septal defect, a ventricular septal defect, and the variations in morphology of the atrioventricular valve or valves (Fig. 15).

\section{References}

Al Omeri, M., Bishop, M., Oakley, C., Bentall, H. H., and Cleland, W. P. (1965). The mitral valve in endocardial cushion defects. British Heart fournal, 27, 161-176.

Bharati, S., and Lev, M. (1973). The spectrum of common atrioventricular orifice (canal). American Heart fournal, 86, 553-561.

Blieden, L. C., Randall, P. A., Castaneda, A. R., Lucas, R. V., jun, and Edwards, J. E. (1974). The 'goose neck' of the endocardial cushion defect. Anatomic basis. Chest, 65, 13-17.

Brandt, P. W. T., Clarkson, P. M., Neutze, J. M., and
Barratt-Boves, B. G. (1972). Left ventricular cineangiocardiography in endocardial cushion defects (persistent common atrioventricular canal). Australasian Radiology, 16, 367-376.

Campbell, M., and Missen, G. A. K. (1957). Endocardial cushion defects: common atrioventricular canal and ostium primum. British Heart fournal, 19, 403-418.

Ebert, P. A., and Goor, D. A. (1978). Complete atrioventricular canal malformation: further clarification of the anatomy of the common leaflet and its relationship to the VSD in surgical correction. Annals of Thoracic Surgery, 25, 134-143.

Frater, R. W. M. (1965). Persistent common atrioventricular canal: anatomy and function in relation to surgical repair. Circulation, 32, 120-129.

Freedom, R. M., Bini, M., and Rowe, R. D. (1978). Endocardial cushion defect and significant hypoplasia of the left ventricle: a distinct clinical and pathological entity. European fournal of Cardiology, 7, 263-282.

Gerbode, F., Sanchez, P. A., Arguero, R., Kerth, W. H., Hill, J. D., deVries, P. A., Seller, A., and Robinson, S. J. (1967). Endocardial cushion defects. Annals of Surgery, 166, 486-495.

Goor, D. A., and Lillehei, C. W. (1975). Atrioventricular canal malformations. In Congenital Malformations of the Heart, p. 132. Grune \& Stratton, New York.

Goor, D., Lillehei, C. W., and Edwards, J. E. (1968). Further observations on the pathology of the atrioventricular canal malformation. Archives of Surgery, 97, 954-962.

Macartney, F. J., Rees, P. G., Daly, K., Piccoli, G. P., Taylor, J. F. N., de Leval, M., Stark, J., and Anderson, R. H. (1979). Angiocardiographic appearances of atrioventricular defects with particular reference to distinction of ostium primum atrial septal defect from common atrioventricular orifice. British Heart fournal, 42, 640-656.

Neufeld, H. N., Titus, J. L., DuShane, J. W., Burchell, H. B., and Edwards, J. C. (1961). Isolated ventricular septal defect of the persistent common atrioventricular canal type. Circulation, 23, 685-696.

Piccoli, G. P., Wilkinson, J. L., Macartney, F. J., Gerlis, L. M., and Anderson, R. H. (1979). Morphology and classification of complete atrioventricular defects. British Heart fournal, 42, 633-639.

Rastelli, G. C., Kirklin, J. W., and Kincaid, O. W. (1967). Angiocardiography of persistent common atrioventricular canal. Mayo Clinic Proceedings, 42, 200-209.

Rastelli, G. C., Kirklin, J. W., and Titus, J. L. (1966). Anatomic observations on complete form of persistent common atrioventricular canal with special reference to atrioventricular valves. Mayo Clinic Proceedings, 41, 296-308.

Rastelli, G. C., Ongley, P. A., Kirklin, J. W., and McGoon, D. C. (1968). Surgical repair of the complete form of persistent common atrioventricular canal. fournal of Thoracic and Cardiovascular Surgery, 55, 299-308.

Rogers, H. M., and Edwards, J. E. (1948). Incomplete division of the atrioventricular canal with patent interatrial foramen primum (persistent common 
atrioventricular ostium): report of 5 cases and review of literature. American Heart fournal, 36, 28-54.

Somerville, J. (1966). Clinical assessment of the function of the mitral valve in atrioventricular defects related to the anatomy. American Heart fournal, 71, 701-711.

Somerville, J., and Jefferson, K. (1968). Left ventricular angiography in atrioventricular defects. British Heart fournal, 30, 446-457.

Sumner, R. G., Phillips, J. H., Jacoby, W. J., jun, and Tucker, D. H. (1967). Forme fruste of endocardial cushion defect. American fournal of the Medical Sciences, 254, 266-283.

Tenckhoff, L., and Stamm, S. J. (1973). An analysis of 35 cases of the complete form of persistent common atrioventricular canal. Circulation, 48, 416-427.

Ugarte, M., Enriquez de Salamanca, F., and Quero, $M$. (1976). Endocardial cushion defects. An anatomical study of 54 specimens. British Heart fournal, 38, 674-682.

Van Mierop, L. H. S. (1977). Pathology and pathogenesis of endocardial cushion defect. Surgical implications. In Second Henry Ford Hospital Inter- national Symposium on Cardiac Surgery, pp. 201-207, ed J. C. Davila. Appleton-Century-Crofts, New York. Van Mierop, L. H. S., and Alley, R. D. (1966). The management of the cleft mitral valve in endocardial cushion defects. Annals of Thoracic Surgery, 2, 416423.

Van Mierop, L. H. S., Alley, R. D., Kausel, H. W., and Stranahan, A. (1962). The anatomy and embryology of endocardial cushion defects. Fournal of Thoracic and Cardiovascular Surgery, 43, 71-83.

Wakai, C. S., and Edwards, J. E. (1956). Developmental and pathologic considerations in persistent common atrioventricular canal. Proceedings of the Staff Meetings of the Mayo Clinic, 31, 487-500.

Wakai, C. S., and Edwards, J. E. (1958). Pathologic study of persistent common atrioventricular canal. American Heart fournal, 56, 779-794.

Requests for reprints to Dr R. H. Anderson, Department of Paediatrics, Brompton Hospital, Fulham Road, London SW3 6HP. 